Lexique vivant de la révolution et de la guerre en Syrie

\title{
Contester l'omnipotence \\ du leader, conjurer et \\ susciter la peur. Les usages \\ multiples de la formule \\ Allâhu Akbar / الله أكبر
}

Emma Aubin-Boltanski ${ }^{1}$,

Séminaire les mots de la révolution et de la guerre en Syrie ${ }^{2}$

${ }^{1}$ Césor, ${ }^{2}$ EHESS

Published on: Jan 25, 2022

License: Creative Commons Attribution 4.0 International License (CC-BY 4.0). 


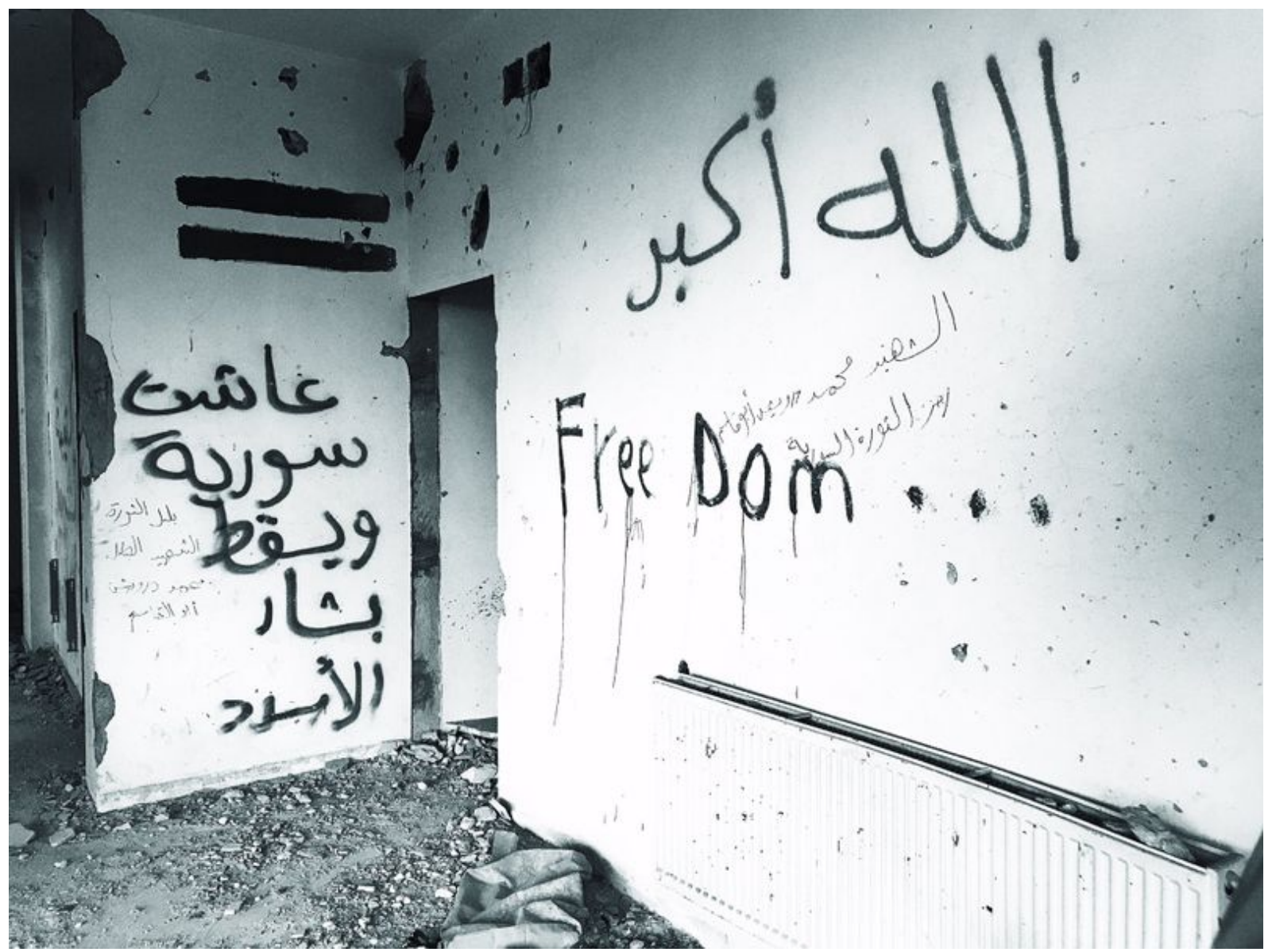

Figure 1. Graffitis de la ville de Rastan. De droite à gauche on peut lire : Allâhu Akbar Free Dom, Vive la Syrie et que tombe Bachar al-Assad https://web.archive.org/web/20211106173212/https://twitter.com/ameralnasser 1/status/882438017025097729?lang=ar

Cet article a été élaboré en 2021 dans le séminaire "Les mots de la révolte et de la guerre en Syrie" coordonné par Nisrine al-Zahre, Emma Aubin-Boltanski et JeanChristophe Peyssard. Les personnes suivantes y ont contribué : Eylaf Bader Eddin, Nibras Chehayed, Rana Diab, Vanessa Guéno, Oussama Khalbous, Charlotte al-Khalili, Boris James, Thomas Pierret, Dommar al-Solemam.

Le takbîr/ou le fait de prononcer la formule Allâhu Akbar/ الله أكبر/Dieu est [le] plus grand ») est devenu dès les premiers jours du soulèvement de mars 2011 en Syrie l'un des slogans des manifestants. Le régime assadien a voulu y voir la preuve que les manifestants étaient des islamistes, et ainsi légitimer sa politique de répression. Dans les médias occidentaux également cette lecture a prévalu, et ce d'autant plus que dans la décennie 2010, des attentats sont commis dans différentes capitales européennes par des jihadistes dont le cri de guerre n'est autre que Allâhu Akbar. Des voix se sont 
élevées pour remettre en cause ces lectures biaisées et rappeler les usages pluriels et le caractère parfois banal de cette expression. " Mon père athée et d'une famille chrétienne disait Allâhu Akbar à tout bout de champ, un peu comme on dit 'enfin !' ou 'super !' en français quand une bonne nouvelle arrive ou quand on est soulagé », explique Sana pour souligner la banalité de la formule. Autre usage, autre contexte : dans la pièce de théâtre Egalité, Omar Abu Michel, le personnage joué par l'acteur et metteur en scène syrien Nawar Bulbul, tente une explication du takbîr en des termes intéressants, car tout à la fois subversifs et révélateurs des malentendus, parfois graves, qui peuvent se produire lorsqu'un Syrien vivant en Occident prononce la formule :

Je suis d'accord avec vous monsieur. 'Allâhu Akbar' chez les autres c'est très dangereux, mais 'Allâhu Akbar' chez les Syriens c'est rien. C'est comme quand vous dites en Français : 'Oh putain de journée de merde !1․

En Syrie, comme ailleurs au Proche-Orient, le takbîr est un élément banal et quotidien du paysage sonore. Ses usages interjectifs - formule religieuse, acclamation, slogan ou exhortation - sont multiples et liés à des émotions aussi différentes que l'enthousiasme, l'exaspération, la peur, l'espoir ou encore la haine de l'autre. À l'instar de « Dieu merci »/ « grand Dieu » en français ou de « thank God »/ « my God » en anglais, certains sont résolument sécularisés alors que d'autres sont clairement religieux et/ou politiques. L'éventail des registres d'actions dans lequel le takbîr s'inscrit est tout aussi large : solennel, religieux, militant, guerrier ou festif $\underline{2}$.

Comment l'engagement politique se débrouille-t-il avec les mots ?, en guise de réponse à cette question, Donna Haraway développe une réflexion qui nous paraît bien s'appliquer au rôle que certaines expressions, - et particulièrement Allâhu Akbar -, ont joué dans le soulèvement syrien : les mots explique-t-elle sont « des forces matérielles sémiotiques, ils remplissent la bouche. Ce sont des créatures de la terre, des entités douées d'une grande force, et elles ne sont pas nécessairement sous contrôle, elles ont une matérialité et une force qui leur est propre. Les mots ne sont jamais simplement des mots, le discours jamais simplement du discours, la textualité jamais simplement de la textualité. Il y a en eux une sorte d'ici-et-maintenant matériel sémiotique. (...) Les mots sont des pouvoirs de faire-monde, ils font des choses $\underline{3}$.

En quelques phrases, Haraway cerne avec justesse plusieurs dimensions qui nous intéressent : la matérialité des mots - qui s'énoncent, s'écoutent, s'inscrivent, sont vus et touchés -, leur lien avec les corps de celles et ceux qui les clament, parfois les 
hurlent, les chantent, les murmurent, les brandissent, les dansent, les jettent à la face du tyran, etc. ; leur autonomie - ce sont des forces positives et/ou négatives qui, ne se laissant pas enfermer dans des définitions uniques, peuvent échapper à ceux-là même qui les énoncent : être à l'origine de hiatus, voire même de malentendus dramatiques.

\section{Contester l'omnipotence du leader}

« Le takbîr marquait le début des manifestations, explique Eylaf Bader Eddin, avant de préciser : un meneur disait 'takbîr' et les manifestants répondaient 'Allâhu Akbar'.

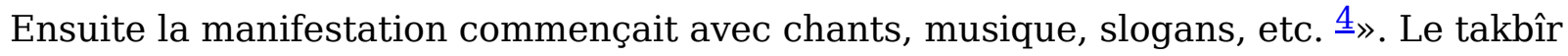
sert dans ce cas précis à « chauffer la foule $\underline{5}$ ». C'est un geste collectif et coordonné accompli pour faire corps, instituer une communauté. Les manifestants clamaient ensemble Allâhu Akbar comme ailleurs des foules crieraient d'une seule voix « hourra » en français ou « hurrah » en anglais, encouragées par un meneur. Pour autant, le caractère religieux, même minoré, de la formule demeure. De plus, l'usage extensif du takbîr dans les manifestations pacifiques des premiers mois du soulèvement est une particularité syrienne que l'on ne retrouve ni sur la scène de la révolution tunisienne, ni non plus en Égypte $\underline{6}$. Comment le comprendre ?

Pour illustrer notre propos, commençons par décrire une vidéo de manifestation 7 . Tournée le 18 décembre 2011 à Homs, on y voit l'actrice Fadwa Suleiman $\underline{8}$, juchée sur un caisson, un micro à la main haranguer des hommes et des femmes rassemblés sur la place Khalidiyya. La foule paraît peu nombreuse, mais l'entrain et la joie sont palpables. «Aujourd'hui, il y a un pèlerinage (hajj) à Homs, un pèlerinage plus grand encore à Hama » commence par annoncer l'actrice. En agitant des drapeaux syriens et en dansant, la foule en liesse scande pendant plusieurs minutes «Allâhu Akbar » au rythme de tambourins. "Aujourd'hui, un pèlerinage de Tartous est en marche vers Homs » reprend l'actrice, avant d'enchaîner d'une voix forte des « Allâhu Akbar huriyya » (Dieu est le plus grand, liberté) répétés par la foule. Dans l'ensemble de la séquence de quatre minutes, outre le takbîr, on relève un grand nombre d'expressions religieuses : Fadwa Suleiman, qui pourtant se définit comme laïque, ne parle pas de « manifestation », mais de «pèlerinage »; à plusieurs reprises la foule lui adresse des «Que Dieu te protège »; enfin pendant quelques minutes elle décline une profession de foi interreligieuse associant la figure de Jésus à celle de Muhammad, mêlant formules musulmane et chrétienne : «Il n’y a de Dieu que dieu, Muhammad est l'envoyé de Dieu et Jésus est le prophète de Dieu. Gloire à Dieu ». Tout en sacralisant la révolte en cours, ces paroles clamées dans une atmosphère à la fois festive et grave disent le désir de rompre avec les convenances, le refus de rester prisonniers des 
normes établies, qu'elles soient religieuses ou politiques. La licence subversive qu'elles s'autorisent - qui consiste à associer des mots et des formules communément considérées comme antinomiques et à ignorer les barrières confessionnelles - sont une forme de « procès de langage » qui appelle à une révision globale du système social et culturel qui a cours.

« Les manifestants ont eu recours au takbîr et à des slogans religieux, pour faire tomber les attributs divins attachés au régime syrien et affirmer l'existence d'un être plus grand et plus haut », explique avec justesse Dommar Al-Soleman $\underline{9}$. Cet usage du takbîr et de formules religieuses a été observé en particulier pendant les premières semaines du soulèvement. Il doit être compris comme une réaction à la « déification » de Hafez al-Assad, puis de son fils, imposée depuis le massacre de Hama de 19821ㅡ. Lors de la confrontation avec les Frères musulmans, des unités militaires et paramilitaires d'élite majoritairement composées d'alaouites s'en prennent à des symboles religieux sunnites. L'idéologie baasiste dominée par des thèses modernistes hostiles à la religion ne permet qu'en partie de comprendre ces actes qui sont avant tout une réaction confessionnelle au retour de l'islam sunnite comme force politique. Dans le même temps, le culte entourant le président est exacerbé. Il en a résulté la mise en place d'une forme singulière d'« idolâtrie profanatrice $\underline{11}$ » qui est tout autant l'expression de la loyauté des partisans au régime que le moyen de profaner la foi en Dieu. Cette idéo-théologie ne vise pas tant à construire une religion civile unificatrice qu'à humilier la foi religieuse des opposants en leur imposant l'idolâtrie du chef et du parti. Pour les philosophes Nibras Chehayed et Guillaume de Vault, elle constitue « un instrument de destruction du sol commun sur lequel pourraient se construire des

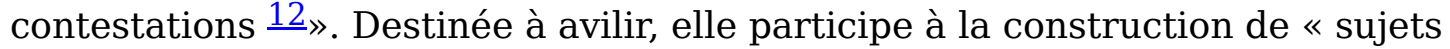
abjects $\gg \underline{13}$.

Revenons sur quelques-uns des actes marquants de cette idolâtrie profanatrice contre laquelle les manifestants brandissent le takbîr. En 1982, lors de l'assaut de Hama des soldats scandaient des slogans tels que : «Ô Dieu il est temps, il est temps que Hafez prenne ta place » (يالله حلك حلك يقعد حافظ محلك). Sur un mur de la ville, on pouvait lire ce détournement de la profession de foi musulmane « Il n'y a de divinité que la nation et il n’y a de prophète que le parti Baas »)(15). لا الله إلا الوطن ولا رسول إلا البعث. 


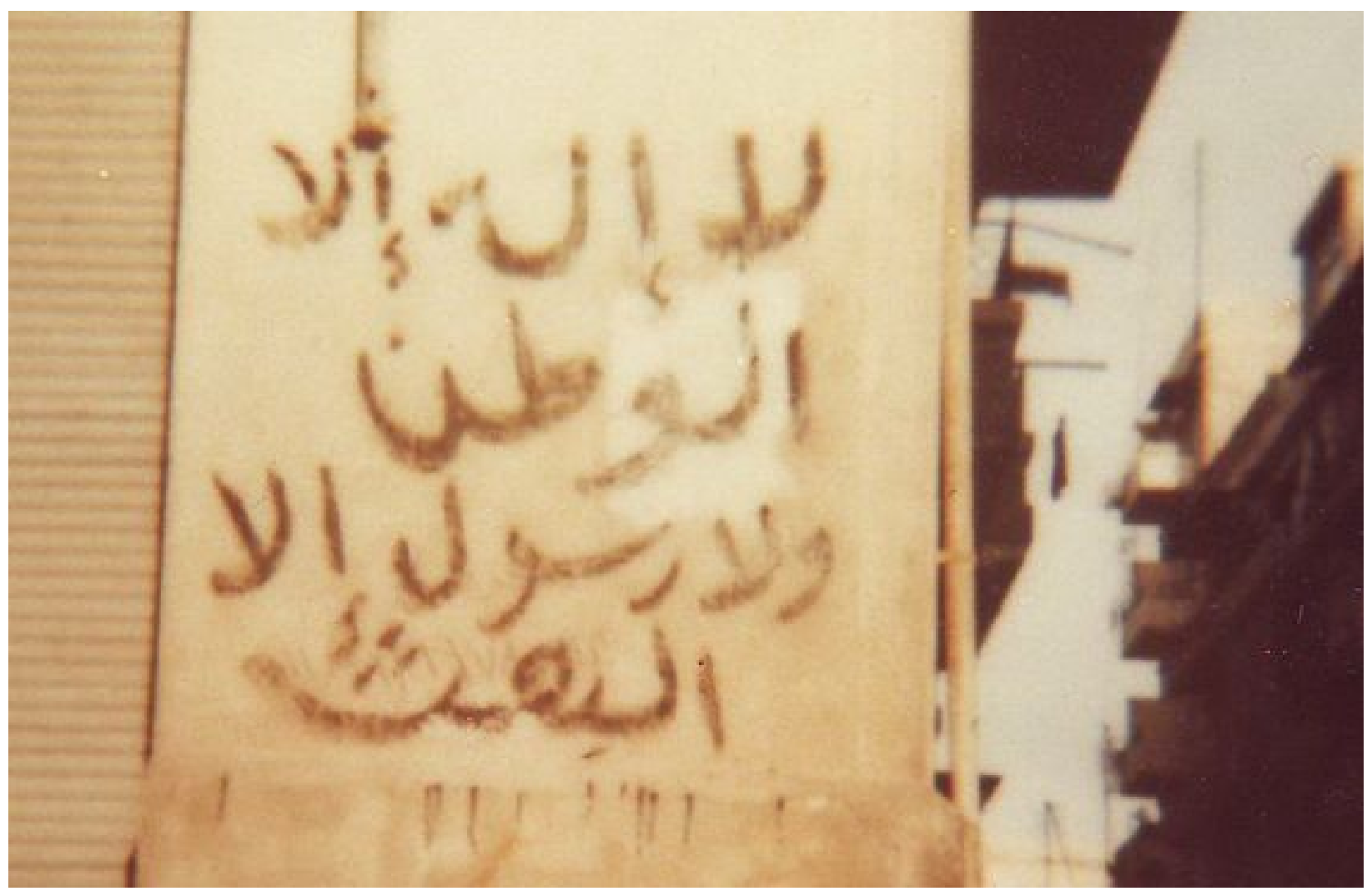

Figure 2. "Il n’y a de divinité que la nation et il n’y a de prophète que le parti Baas ». Ce graffiti fut inscrit sur un mur de la ville de Hama en 1982. Capture d’écran du film Suwwar jadîda 'an majzarat hama 1982 [Nouvelles images sur le massacre de Hama 1982], YouTube, mis en ligne le 5 août 2011, https://www.youtube.com/watch?v=qexuAYba3xU

Pendant les décennies qui ont suivi les évènements de Hama, la tendance irréligieuse du régime a été moins ostentatoire, mais dès les premiers jours du soulèvement de 2011, des vidéos de minarets pris pour cibles par l'armée du régime à Homs et à Deir ez-Zor $\underline{16}$ circulent sur Internet. Ces attaques injustifiées militairement sont interprétées comme l'expression d'une « haine » à l'égard de l'islam $\underline{17}$. Certains y voient une réaction aux déboulonnages de statues de Hafez al-Assad qui se généralisent dans le pays depuis mars 2011트. Dans la même période, les slogans scandés par les Minhabakjî (litt. les « Nous-t'aimons-istes », néologisme qui désigne les partisans de Bachar al-Assad) ont une forte tonalité sacrilège. «Ô Dieu bouge-toi de là et laisse Bachar prendre ta place $\gg \underline{19}$, à cette reprise de 1982 , s'ajoutent de nouvelles formules plus idolâtres encore : «Bachar : là où tu poses ton pied, nous nous agenouillons et nous embrassons [le sol]»(بق) (بشار: مطرح ما بتدوس بنركع و منبوس. En réaction au slogan des manifestants «Le peuple veut la chute du régime », les mêmes répondent : 《Que ton seigneur tombe, pas al-Assad» (بسقط ربك وما بسقط الأسد). En juillet 2011, apparaissent dans les rues de Damas des bannières sur lesquelles des 
قل هو : photos des Assad père et fils encadrent sur un fond bleu nuit étoilé la formule (Dis : "Il est Allah, unique. Allah, Le Seul à être imploré pour ce que nous désirons. Dieu exalte mon peuple et a élu parmi lui le

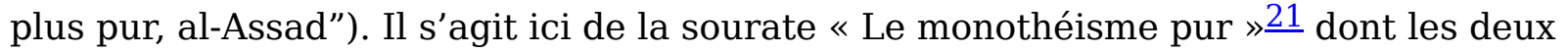
derniers versets ( «Il n’a jamais engendré, n’a pas été engendré non plus et nul n’est égal à lui ! ») ont été subvertis pour signifier la gloire quasi prophétique d'al-Assad $\underline{22}$.

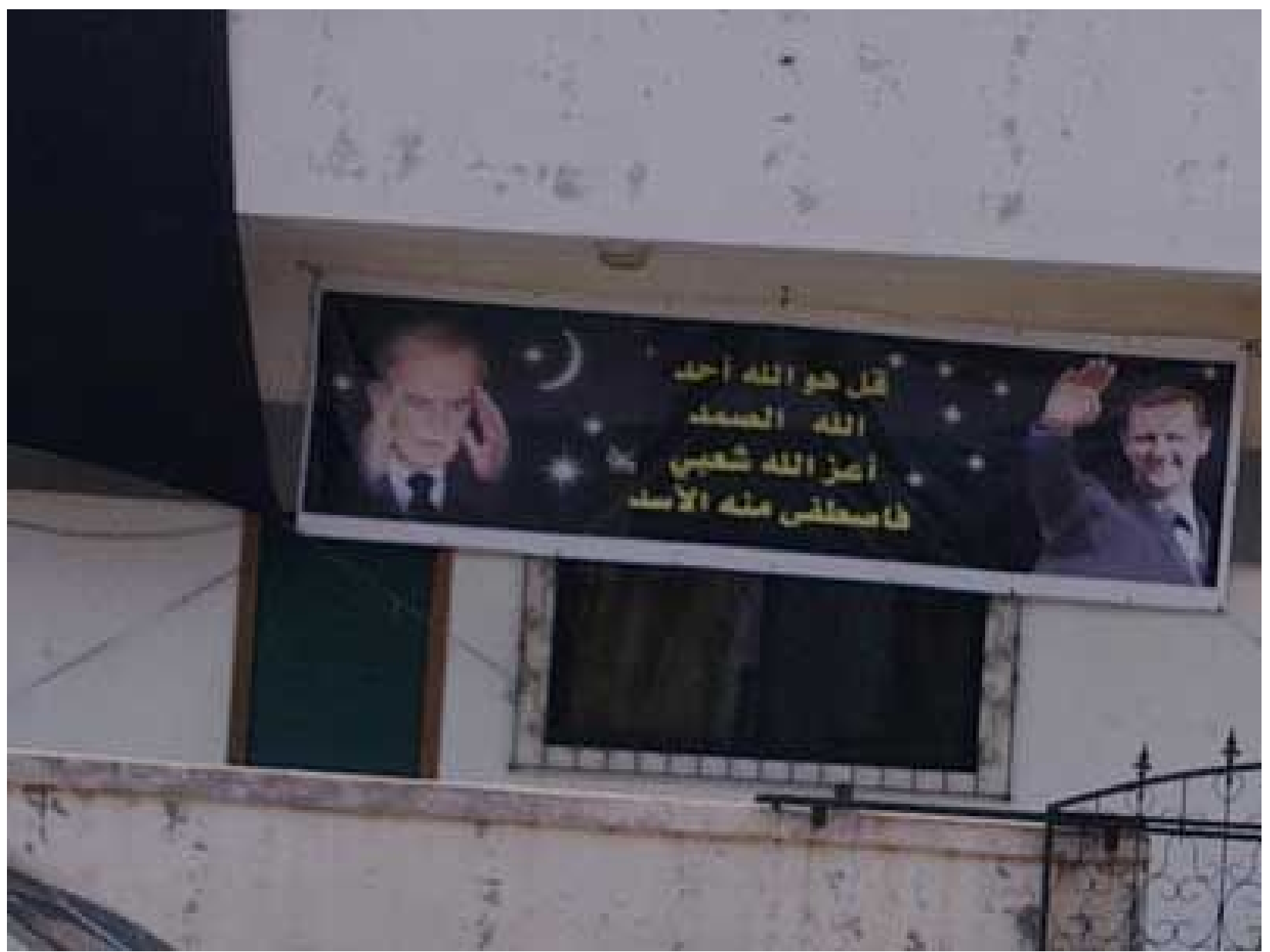

Figure 3. Bannière sur laquelle on peut lire: "Dis "Il est Allâh, unique. Allâh, Le Seul à être imploré pour ce que nous désirons. Dieu exalte mon peuple et a élu parmi lui le plus pur, al-Assad"", source : Zamanalwsl https://www.zamanalwsl.net/news/article/20371/

L'idolâtrie profanatrice de la personne du président va jusqu'à devenir une forme de torture exercée par les nervis du régime à l'encontre des opposants. Sur une vidéo filmée par des soldats probablement en 2011, un homme assis au sol, les yeux bandés est contraint de répondre «Bachar al-Assad » à chacune des questions qui lui sont hurlées aux visages : «Qui est ton Dieu ? 》; «Qui pries-tu ? » et doit finir par clamer à gorge déployée : «Il n’y a de divinité que Bachar » $\underline{23}$. Des détenus auraient été 
sommairement exécutés après avoir refusé de se prosterner devant un portrait d'alAssad en professant qu'il était leur Dieu는. Déjà dans les années 1980, de tels actes ont été commis : Le poète Faraj al-Biraqdar évoque dans ses mémoires de prison sa rencontre avec un geôlier qui après lui avoir demandé ce qu'il n'aimait pas dans Hafez al-Assad, lui aurait affirmé que l'urine du leader était curative et que ses excréments devaient être transformés en sanctuaire $\underline{25}$.

Lors d'un discours prononcé en août 2011 devant une assemblée d'oulémas Bachar alAssad commente sur un ton détaché et débonnaire ces différentes affaires (tirs sur les mosquées, idolâtrie profanatrice imposée aux détenus). Selon lui, il s'agit d’actes « impies » et donc « condamnables », mais « isolés », ne pouvant être attribués au service de sécurité ou à l'armée dans leur ensemble. En résumé, il nie l'existence d'une stratégie de provocation à l'égard des musulmans sunnites $\underline{26}$. De fait, ces actes de provocations qui se multiplient $\underline{27}$ ne paraissent pas relever d'une politique délibérée du régime. Ils sont probablement l'expression spontanée d'une sous-culture anti-sunnite profondément ancrée chez des membres alaouites des forces armées. Parce qu'ils risquent de lui aliéner les segments les plus loyaux de la communauté sunnite (certains hommes d'affaires et religieux), Bachar al-Assad choisit de les condamner.

Ainsi donc, à l'instar de Fadwa Suleiman, en mars 2011, contre l'idolâtrie profanatrice imposée par le régime, des femmes et des hommes, croyants ou non, sont descendus dans la rue pour clamer d'une seule voix le takbîr et en quelque sorte hurler à la face du leader omnipotent l'existence d'une autre forme d'absolu. Le Allâhu akbar est devenu l'un des termes du lexique de l'engagement révolutionnaire en raison de la présence diffuse de l'islam sunnite majoritaire dans la culture et le paysage sonore quotidien des Syriens. Mais pas seulement : pour celles et ceux qui le clamaient, la charge sémantique de chacun des mots comptait. Dans son étude sur les cercles religieux sunnites de Daraya, Charlotte al-Khalili explique que déjà dans les années 2000, faire allégeance au leader de la nation et proclamer le principe de l'unicité divine étaient perçus comme des gestes langagiers contradictoires $\underline{28}$. En 2011, invoquer Dieu, en appeler à sa transcendance en l'associant au désir de liberté devient un acte de désobéissance, une façon d'exprimer son refus de se soumettre pour des personnes qui pour certaines étaient incroyantes $\underline{29}$.

Dans les manifestations des premiers mois de la révolution syrienne (le rassemblement autour de Fadwa Suleiman en est un exemple) l'énonciation du takbîr est accompagnée sans la conjonction de coordination « et » du mot huriyya (liberté) pour 
composer une seule acclamation : « Dieu est le plus grand liberté ». Ce takbîr augmenté devient le refrain d'une chanson de la révolution $\underline{30}$.

« Le nom de Dieu a été par là transformé en lieu de liberté », explique Nibras Chehayed dans un article publié en janvier 2011 dans le journal Assafir intitulé «Quand il ne reste plus qu'aux Syriens le nom de Dieu » $\underline{31}$. Le philosophe s'interroge sur ce « nom » présent dans toutes les bouches, celles des croyants, mais aussi celles des athées (mulhidûn) et des agnostiques (lâ-adriyûn). Il avance l'hypothèse que ce nom constitue une blessure dans le corps du langage lorsqu'il est pris au sérieux, autrement dit lorsqu'il renvoie à un sujet qui se dérobe par principe. Dès lors, parler de Dieu c'est aussi parler de ce que Dieu n'est pas, ce qui fait appel à une déconstruction permanente des discours religieux et areligieux au nom de Dieu luimême. C'est dans cette perspective que ce « Dieu linguistique » peut devenir synonyme d'espérance politique pour tous les Syriens, mais à condition de demeurer le lieu d'une liberté pour tous et de ne pas se laisser enfermer dans une définition unique et exclusive. Bien que fragile et menacée, cette acception ouverte du nom de Dieu est, selon Nibras Chehayed, bien présente sur la scène de la révolution en 2011. Effectivement, la profession de foi interreligieuse scandée par Fadwa Souleiman et reprise par la foule des manifestants à Homs en est un exemple saisissant.

\section{Conjurer la peur}

Nous l'avons dit, le champ des émotions exprimées par le takbîr est vaste. Sur la scène de la révolution, il semble en exprimer deux en particulier : l'enthousiasme d'une part et la peur de l'autre. « On criait et on répétait Dieu est le plus grand pour s'encourager mutuellement, pour arrêter d'avoir peur », explique une activiste. De fait, si l'exaltation suscitée par l'évènement est bien là, renforcée par le fait d'être porté par ces foules immenses qui battent le pavé, dansent, chantent, hurlent ensemble leur désir de justice, de liberté et de dignité, la peur ne disparaît pas tout à fait. Et à raison. Nous avons décrit l'entrain et la joie qui semblaient régner lors du rassemblement du 18 décembre 2011 à Homs autour de Fadwa Suleiman. En réalité, ces manifestants étaient certainement terrorisés. Quelques mois plus tôt, le 18 avril sur la place de l'Horloge située à deux kilomètres de là, un rassemblement avait été pris d'assaut par les forces du régime. Le bilan de ce massacre reste flou, la rumeur évoque plusieurs centaines de morts $\underline{32}$, mais ces chiffres ont été probablement gonflés à des fins de mobilisation. Quoi qu'il en soit, la violence de la répression a provoqué un immense effroi dans la population $\underline{33}$. Ces hommes et ces femmes qui répétaient à gorge 
déployée et en dansant les slogans de l'actrice avaient conscience du risque qu'ils encouraient, celui de mourir.

Manifester et périr sont si étroitement associés que le 9 avril 2011, dans la ville de Banyas, des hommes battent le pavé revêtus de leurs linceuls blancs (akfân) $\underline{34}$, signifiant par ce geste qu'ils sont prêts à mourir ou plus précisément que « déjà morts » plus rien ne peut les atteindre. Ce jour-là, dans la foule compacte, des Allâhu Akbar fusent sporadiquement parmi les slogans appelant à la chute du régime et à la liberté. Ici, le rappel de la transcendance absolue du Dieu unique évoque la prière mortuaire dont il constitue un élément essentiel : dans la tradition sunnite, le takbîr est prononcé quatre fois sur la dépouille de tout musulman, dans la tradition chiite il est répété cinq fois sur celle d'un simple fidèle et sept fois sur celle d'un descendant du Prophète $\underline{35}$.

Dans son journal intime, Nisrine al-Zahre fait le récit de la sanglante répression du sitin de la place de l'Horloge à Homs en avril 2011 et ce qu'elle rapporte est effectivement terrifiant :

La nuit du rassemblement de la place de l'horloge : tout le monde s'entraidait, distribuait la nourriture et l'eau. À l'époque, les Homsiotes étaient encore naïfs, on pensait que le rassemblement allait durer jusqu'à la chute du régime, à l'instar de l'Égypte. À onze heures du soir, les jeunes de la place de l'Horloge ne savaient pas ce qui les attendait, ils échangeaient des idées comme si tout allait vers un mieux. Vers deux heures du matin, les monstres sont arrivés, on ne connaît toujours pas leur identité, car c'est la règle en Syrie, tout doit être mêlé pour être vide de sens, pour que le sens s'évapore avec les âmes rendues des gens (...) Quelques dizaines d’hommes armés habillés en noir ont commencé à tirer sur les gens. La terreur a envahi la ville. Jusqu'à maintenant, on ne connaît pas le nombre exact des tués pendant ce massacre, on rapporte comme chiffres entre deux cents et trois cents personnes. Les habitants du voisinage de la place confirment une seule chose : à l'aube, les pompiers sont venus laver le sol du sang qui remplissait l'air de Homs de l'odeur de la mort. Ils ne peuvent pas donner d'autres détails pour la simple raison que celui qui sortait sa tête par la fenêtre était tué par les francs-tireurs. D'autres habitants parlent de camions de déchets qui ont entassé les corps pour s'en débarrasser quelque part.

Cette nuit-là, poursuit Nisrine al-Zahre, des Allâhu Akbar ont retenti, lancés des mosquées de la ville : 
Le régime a utilisé ce prétexte pour attaquer avec plus de dureté, sa version officielle étant l'existence de salafistes fanatiques à Homs. Je me demande, moi, née d'une famille chrétienne et laïque, ce que j'aurais pu dire d'autre devant cette terreur ? Chez les Syriens, la formule « Allâh Akbar », littéralement « Dieu est le plus grand » revêt un autre sens et signifie qu'il existe certainement un absolu quelconque plus grand que cet absolu du mal !!

Conjurer la peur et, dans le même mouvement, signifier la possibilité d'un « absolu plus grand » que le dictateur omnipotent, tels sont les deux principales fonctions du takbîr qui apparaissent dans une seconde vidéo $\underline{36}$. Filmée également à Homs, mais quelques mois plus tard, en mars 2012, elle est destinée à témoigner de bombardements du régime visant des habitations et des civils. Elle ne dure qu'une minute trente. Caméra à l'épaule, un preneur d'image suit un activiste dans les rues désertées du quartier al-Hamadiyeh ciblé par des tirs de missile. L’homme décrit la situation d'une voix saccadée et apeurée. Les tirs s'intensifient, il faut courir. Tout en continuant à filmer, le cameraman lance sur un ton rageur : " Sois maudit Bachar, Dieu est plus grand que toi !» (الله أكبر عليك يا بشار). L'activiste, quant à lui, répète d'une voix toujours plus terrifiée une vingtaine de fois le takbîr. Ici l'homme a recours à la fonction «miraculeuse » de la formule : il la réitère tel un mantra destiné à le protéger $\underline{37}$.

\section{Susciter la peur chez l'autre}

Pendant les premiers mois du soulèvement syrien - le rassemblement de Homs autour de Fadwa Suleiman en est une illustration -, on observe un usage irénique du Allâhu Akbar, devenu en quelque sorte synonyme de liberté et d'espoir. Toutefois, dans la même période, le takbîr est également brandi comme une menace adressée au pouvoir et plus largement aux membres de la communauté alaouite perçus comme les indéfectibles soutiens du régime.

L'une des actions conduites par les révolutionnaires a, par exemple, consisté à inviter les habitants des centres urbains à monter sur les toits à une heure précise de la nuit pour clamer Allâhu akbar. L'objectif était d'occuper l'espace sonore dans un moment où, la répression s'intensifiant, l'espace physique devenait inaccessible. Cette idée a été directement soufflée par le prédicateur salafiste Adnan al-Ar'ur $\underline{38}$ dans son émission du 25 mai $2011 \underline{39}$. « Les Allâhu akbar résonnaient dans toute la ville de Douma, ponctués occasionnellement par des tirs de kalachnikovs », raconte Oussama Khalbous avant de préciser que l'objectif des révolutionnaires était double : d'une part, 
sur le modèle des manifestations iraniennes de $2009 \underline{40}$, encourager les gens à se rebeller et d'autre part susciter la peur chez les hommes de la Sûreté :

Tu noteras que le takbîr est devenu un slogan en Syrie et pas en Égypte alors que là-bas aussi les Frères musulmans sont très puissants. Pourquoi ? Parce qu'en Syrie même si les gens ne veulent pas le dire, l'expression a une fonction confessionnelle. Les sunnites croient que les alaouites ont peur du Allâhu Akbar et d'ailleurs les alaouites ont effectivement peur d'entendre le Allâhu Akbar. À Douma, lorsque le couvre-feu était décrété, que les gens n'avaient pas le droit de sortir... on se mettait d'accord pour crier le takbîr à la même heure. Et lorsque les forces de sécurité entendaient toutes ces voix dirent le takbîr de l'intérieur des maisons, ils prenaient peur et se mettaient à tirer comme des fous dans le vide, tellement ils avaient peur. Ils entendaient « Suriyya huriyya » (Syrie liberté), ça ne leur faisait rien. Mais le Allâhu akbar, ça leur faisait peur. Le takbîr éveille la peur. Les gens ne disaient pas on va venir vous étrangler. Ils criaient le takbîr et c'était tout comme. Le Allâhu Akbar, c'était comme un code $\underline{41}$.

Le takbîr comme menace destinée à susciter la peur, la formule est de fait, reçue comme telle par nombre de Syriens qu'ils soient minoritaires (alaouites, chrétiens ou autres), laïcs, ou encore sunnites. Dès lors, son usage divise: il tend à réifier les frontières confessionnelles et à provoquer des réflexes de replis communautaires. Dans 19 femmes, recueil de témoignages rassemblés par la romancière Samar Yazbek, Leila, une jeune femme, pourtant elle-même favorable à la révolution, racontent la terreur ressentie par sa famille alaouite vivant dans le quartier de Karm al-Zeitun à Homs lorsque des Allâhu Akbar retentissent dans la ville peu après le massacre de l'Horloge. Pour les manifestants endeuillés, le takbîr a ici une fonction de dénonciation et d'alerte, mais des habitants alaouites de la ville le perçoivent comme une menace qui leur est adressée:

Pendant le sit-in de Homs d'avril 2011 j'ai eu des échanges tendus et difficiles avec ma famille. Je voulais comprendre ce qui se passait. J'étais à Damas quand ma sœur m'a appelée à quatre heures du matin pour me faire entendre des voix qui criaient Allâhu Akbar. Elle était terrifiée. Elle m’a dit : « Des gens hurlent Allâhu Akbar dans les mosquées, ils appellent au jihâd. Ils vont nous tuer ». Le takbîr effraie les alaouites, parce qu'il est lié à la mémoire des pogroms du passé. C'est à ce moment décisif qu'une peur existentielle s'est emparée de mes proches $\underline{42}$. 
En note de bas de page, Samar Yazbek précise que cette anxiété, entretenue par la propagande du régime, s'inscrit dans l'histoire longue de la communauté alaouite longtemps persécutée : «Dans les récits qui se transmettent oralement de génération en génération, on rapporte que des pogroms étaient précédés par des appels au meurtre et des takbîr lancés à partir des mosquées » $\underline{43}$.

Dans un second témoignage, Douha Achour, une ancienne militante du Parti de l'Action communiste, s'attarde également sur l'inquiétude suscitée par le takbîr non pas uniquement chez les alaouites, mais chez l'ensemble des minoritaires syriens, chrétiens, druzes compris :

La vitesse avec laquelle le slogan exigeant la chute du régime est apparu m'a effrayée. J'hésitais à m'impliquer dans un mouvement que je ne comprenais pas, surtout après une expérience d'un quart de siècle dans la politique. Je voyais la panique sur les visages des gens. De nombreux alaouites se sont repliés dans leurs villages, et deux mois seulement après le début de la révolution, mes connaissances chrétiennes et druzes étaient terrifiées. Mes amies druzes ont mis le voile par précaution. Pour elles, qui entendaient des manifestants crier « Allâhu Akbar », c'était une révolution islamiste $\underline{44}$.

« Pour s'assurer qu'ils ne participaient pas à un mouvement religieux ou islamiste, des activistes ont entrepris de subvertir la formule par un jeu de mots, en transformant 'takbir' en 'take a beer' » explique Eylaf Bader Eddin $\underline{45}$. Certes, il s'agit là d'un « trait d'humour », mais cette subversion est aussi révélatrice d'une inquiétude qui gagne les rangs révolutionnaires, car « même si les manifestants ne l'utilisent par pour des raisons islamiques, le takbîr est indéniablement enraciné dans l'islam $\underline{46}$ ». Son emploi, même irénique, est à l'origine de hiatus et de malentendus dramatiques, au sein de la population syrienne, comme sur la scène internationale où dans la décennie 2010, il en vient à être de plus en plus associé au terrorisme islamique. Habilement, le régime a su instrumentaliser la charge négative de l'expression pour justifier la brutalité de sa répression. «Après tout ce qui s'est passé, mon père décédé n’est plus là pour en témoigner, mais c'est sûr, il ne dirait plus Allâhu Akbar à tout bout de champ. Maintenant, même pour les Syriens l'expression n'a plus rien de banal », conclut Sana.

En effet, à partir de 2013, pour une majorité de Syriens, sunnites également, le takbîr en vient à être associé au système de terreur instauré par l'État islamique dans les régions qu'ils contrôlent. Un article paru sur le site arabe de la $\mathrm{BBC} \stackrel{47}{ }$, évoque le cas d'une jeune-fille originaire de la ville d'Azaz (nord-ouest d'Alep) qui, traumatisée par la guerre, dit avoir développé une phobie du Allâhu Akbar: «Quand j'entendais ces mots, 
j'étais prise de frissons, même lorsque c'était ma mère qui les prononçait ou encore lorsque c'était l'appel à la prière de l'aube. Je me réveillais en frissonnant et en suffoquant. Pour moi, ces mots déclenchaient des tirs, des explosions, des hurlements de femmes et d'enfants (...). Ils étaient associés à des hommes cruels qui égorgeaient, tuaient, kidnappaient, brûlaient et violaient des femmes sans pitié ».

\section{Comment traduire Allâhu Akbar?}

Pour conclure, quelques mots s'imposent sur l'épineuse question de la traduction du takbîr en anglais et en français. Dans sa thèse de traductologie, Eylaf Bader Eddin consacre plusieurs pages à l'analyse des choix opérés par Max Weis pour traduire en anglais des slogans révolutionnaires mentionnés dans Feu croisé. Journal de la révolution syrienne de Samar Yazbek. Concernant Allâhu Akbar, il critique avec raison la stratégie de « dépaysement » (ou forainisation) $\underline{48}$ du traducteur qui consiste à translittérer en lettre latine la formule :

The first day Jableh started calling out Allâhu Akbar from inside the houses, the security and the shabbiha began treating people brutally. The security response was awful and very violent $\underline{49}$.

Ce choix est en effet problématique, car il peut conduire à des malentendus, le Allâhu Akbar (non traduit) étant étroitement associé aux attaques terroristes islamistes dans les imaginaires occidentaux. La stratégie inverse consisterait à « domestiquer » dans la langue cible la formule, en prenant en compte le contexte précis d'énonciation pour chaque occurrence. Ce que propose Eylaf Bader Eddin. Parce que le takbîr, dans la situation rapportée par Samar Yazbek datée du 21 mai 2011, marquait le début des manifestations pacifiques il suggère une «domestication » radicale : " Hey let's demonstrate ! » $\underline{50}$. Cette proposition a le mérite de rendre compte de la dimension profane, banale, presque légère de l'expression, mais en choisissant de démonétiser le caractère politico-religieux de la formule, elle ne permet pas de comprendre pourquoi les nervis du régime réagirent avec une telle violence lorsqu'ils entendirent le takbîr résonner dans la ville de Jabla, ni non plus pourquoi c'est « de l'intérieur des maisons » que les rebelles parfois terrifiés scandaient la formule.

\section{Bibliographie}

La bibliographie de cet article est disponible dans le groupe Zotero du Lexique (https://www.zotero.org/groups/4550572/syria lexicon/collections/YJC3KUKJ). 


\section{Footnotes}

1. Nawar Bulbul, Égalité, création de la compagnie la Scène-Manassa, 2021, https://vimeo.com/538378921

2. Pour une comparaison avec l'interjection «mon Dieu », cf. Olivier, Claudine.

«L'interjection mon Dieu : variabilité sémantique et situations de discours ». Cahiers de praxématique, $\mathrm{n}^{0} 34$ (1 janvier 2000): 161-89.

https://doi.org/10.4000/praxematique.434.

3. Caeymaex, Florence, Vinciane Despret, et Julien Pieron. « Le rire de Méduse.

Entretien avec Donna Haraway ». In Habiter le trouble avec Donna Haraway.

Editions Dehors, 2019. https://orbi.uliege.be/handle/2268/206424. dans Haraway, Donna Jeanne, Florence Caeymaex, Vinciane Despret, et Julien Pieron. Habiter le trouble avec Donna Haraway. Bellevaux: Éditions Dehors, 2020.

http://www.worldcat.org/oclc/1281882609..

4.

Note Eylaf Bader Eddin dans sa thèse p. 264 :

Bader Eddin, Eylaf. « Translating the language of the Syrian Revolution (2011/12) ». http://www.theses.fr. Thesis, Aix-Marseille, 2020.

http://www.theses.fr/2020AIXM0546.

5. Leyla Dakhli et al. « Allah akbar », dans Dakhli, Leyla. L'esprit de la révolte : archives et actualité des révolutions arabes, 2020.

http://www.worldcat.org/oclc/1220977041. p. 159-163. En ligne : DREAM. « «Allah Akbar » ». Consulté le 5 janvier 2022.

https://dream.hypotheses.org/publication/lesprit-de-la-revolte/produire/allah-akbar.. 6. Pour les jeunes activistes tunisiens, user du takbir devient même un geste de solidarité avec les révolutionnaires syriens, Leya Dakhli et al., op. cit., p. 161. 7.

Homs hayy al-khâlidiyya wa kalima râ'i’a li-l-fannâna al-thâ'ira Fadwa Suleiman 18 122011 [Homs quartier d'al-Khalidiyya le discours génial de la révolutionnaire Fadwa Suleiman 18/12/2011] :

تنسيقية حي الخالدية بحمص. حمص حي الخالدية وكلمة رالائعة للفنانة الثائرة فدوى سليمان 1812 2011 ,2011. https://www.youtube.com/watch?v=vis3yKBqkuY. 
8. « Fadwa Souleimane ». In Wikipédia, 3 octobre 2021.

https://fr.wikipedia.org/w/index.php?title=Fadwa Souleimane\&oldid=186838662. 9. Dommar Al-Soleman, étudiant master 2 , « les raisons de la violence généralisée dans le quartier Al-Zahra - Homs »

10. Ismail, Salwa. The Rule of Violence: Subjectivity, Memory and Government in Syria. $1^{\text {re }}$ éd. Cambridge University Press, 2018.

https://doi.org/10.1017/9781139424721. p. 32-41.

11. Chehayed, Nibras, et Guillaume de Vaulx d'Arcy. La destructivité en oeuvres: essai sur l'art syrien contemporain. PIFD 298. Beyrouth: Presses de l'Ifpo, 2021. http://www.worldcat.org/oclc/1272095476. p. 14.

12. Ibidem. $\_$

13. Salwa Ismail, op. cit, p. 32-41.

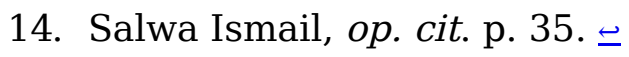

15.

A ce sujet voir le film Suwwar jadîda 'an majzarat Hama 1982 [Nouvelles images sur le massacre de Hama 1982] :

MegaSyrianews. صور جديده تنشر لأول مرة عن مجزرة حماة 1982, 2011. https://www.youtube.com/watch?v=qexuAYba3xU. 16.

Qasf mi'dhana fî bâb al-sabâ' bi-Homs 21-07-2011 [Bombardement du minaret de Bab al-Sabâ' à Homs 21-07-2011] :

المثنى الأحمد. - قصف مئذنة في باب السباع بحمص 21-7-21-2011, 2011. https://www.youtube.com/watch?v=tPzuuiV_JHY.

Deir Ez-zûr qasf wa tadmîr mi'dhanat jâmi' Ibn 'Affân min qabl 'isâbatât al-Assad 1008-2011 [Deir Ez-Zor bombardement et destruction du minaret d'Ibn 'Affân des milices d'al-Assad 10-08-2011], YouTube, mis en ligne le 11 août 2011

ASHONEWS. 2011810 دير الزور قصف و تدمير مئذنة جامع عثمان /بن عفان من قبل عصابات الأسد 2011,2 . https://www.youtube.com/watch?v $=\mathrm{mKNy}$ 448p1N8. 
17. Qasf mi'dhana fî bâb al-sabâ' bi-Homs 21-07-2011 [Bombardement du minaret de Bab al-Sabâ' à Homs 21-07-2011], YouTube, mis en ligne le 22 juillet 2011 https://www.youtube.com/watch?v=tPzuuiV_J on entend en voix off un homme s'exclamer : «C'est de la haine à l'égard de Dieu ». 18.

Isqât timthâl Hafez al-Assad wa samnihi fî madînat Rastan [Chute de la statue de Hafez al-Assad et de son culte dans la ville de Rastan] :

arabsacker. اسقاط تمثال حافظ الأسد وصنمه في مدينة الرستن, 2011. https://www.youtube.com/watch?v=pbdteAvkDl4.. 19. Eylaf Bader Eddin, op. cit. p. 182. 20.

Anas Kamikase « Hafez yahkum min qabrihi » [Hafez gouverne de sa tombe] : / Kebreet (blog), 3 août 2011. https://web.archive.org/web/20211107130034/https://kebreet.wordpress.com/2011/0 8/03/\%D8\%AD\%D8\%A7\%D9\%81\%D8\%B8-\%D9\%8A\%D8\%AD\%D9\%83\%D9\%85\%D9\%85\%D9\%86-\%D9\%82\%D8\%A8\%D8\%B1\%D9\%87/. 21. Sourate 112, al-Ikhlâs (Le monothéisme pur). https://coran1221.org/fr/sourates/s112

22.

Lâfitât tuharrif al-qur'ân al-karîm karma li-‘uyûn al-ra'îs al-sûrî [Des bannières falsifient le Coran pour les yeux du Président syrien] :

《 \ Consulté le 5 janvier 2022. https://web.archive.org/web/20200812134903/https://www.zamanalwsl.net/news/artic $\underline{\mathrm{le} / 20371 / .}$.

23. لا إله إلا بشار الأسد. Consulté le 5 janvier 2022. https://www.facebook.com/watch/? $\mathrm{v}=569549369870949$.

24. Curtis, Peter Theo. «Peter Theo Curtis's Writing on The Twisted, Terrifying Last Days of Assad's Syria ». The New Republic, 4 octobre 2011.

https://web.archive.org/web/20210126135613/https://newrepublic.com/article/95722/ syria-damascus-bashar-basil-al-assad-sunni-alawi. 
25. Cité dans Salwa Isma'il, op. cit., p. 36.

26.

Bachar al-Assad yatahaddath 'an qasf al-mi'dhana wa man qâla lâ ilâh illâ bashâr [Bachar al-Assad parle du bombardement du minaret et de celui qui a dit 'Il n'y a de Dieu que Bachar] :

ExtraPcForum. بشار الاسد يتحدث عن قصف المئذنة ومن قال لا إله الا بشار, 2011. https://www.youtube.com/watch?v=Bz1IwXHhrxI.. 27.

Sûrî yu'abbir 'an ghadabihi : fî-zill al-Assad, narâ al-tawâtul 'an al-Dîn kull yawm [Un Syrien exprime sa colère : Sous al-Assad, nous voyons des attaques contre la religion tous les jours] :

فرانس 24 - مراقبون. 》 سوري يعبر عن غضبه: “في ظل الأسد، نرى التطاول على الدين كل يوم” «, 25 septembre 2012.

https://web.archive.org/web/20211107142301/https://observers.france24.com/ar/201 20925-\%D8\%B3\%D9\%88\%D8\%B1\%D9\%8A-\%D9\%8A\%D8\%B9\%D8\%A8\%D8\%B1\%D8\%B9\%D9\%86-\%D8\%BA\%D8\%B6\%D8\%A8\%D9\%87-\%D9\%81\%D9\%8A\%D8\%B8\%D9\%84-\%D8\%A7\%D9\%84\%D8\%A3\%D8\%B3\%D8\%AF\%D8\%8C\%D9\%86\%D8\%B1\%D9\%89\%D8\%A7\%D9\%84\%D8\%AA\%D8\%B7\%D8\%A7\%D9\%88\%D9\%84\%D8\%B9\%D9\%84\%D9\%89-\%D8\%A7\%D9\%84\%D8\%AF\%D9\%8A\%D9\%86\%D8\%A8\%D8\%B1\%D8\%A7\%D8\%A1\%D8\%A9\%D8\%A7\%D9\%84\%D9\%85\%D8\%B3\%D9\%84\%D9\%85\%D9\%8A\%D9\%86. 28. Al-Khalili, Charlotte. «Halaqas, Relational Subjects, and Revolutionary Committees in Syria ». Focaal 2021, nº 91 (1 décembre 2021): 50-66. https://doi.org/10.3167/fcl.2021.910104. p. 50-56 29. Boëx, Cécile, et Paulo Pinto. « Restituer la densité et la diversité des liens entre le religieux et le politique ». Archives de sciences sociales des religions, $\mathrm{n}^{0} 181$ (1 mars 2018): 11-23. https://doi.org/10.4000/assr.38502. p. 11-23. 30. 
Ughniyya râ'i‘a : Allahu Akbar huriyya. Muzâharât Zamlakâ 10-08-2011 [Une chanson géniale. Allahu Akbar huriyya. Manifestation de Zamlakâ 10-08-2011] :

أبو الفداء الحموي. أغنية رائعة : الله أكبر حرية - مظاهرات زملكا 20-8-2011, 2011 https://www.youtube.com/watch?v=DGl3KdOXs0U. 31.

Nibras Chehayed, " 'indama lâ yabqâ li-l-sûriyyîn ghayr ism Allâh » [Quand il ne reste plus qu'aux Syrien le nom de Dieu], publié dans assafir le 21 juillet 2012, consultable sur le site Kebreet :

Kebreet (blog), 22 janvier 2012.

https://web.archive.org/web/20211107140411/https://kebreet.wordpress.com/2012/0 1/22/\%D8\%B9\%D9\%86\%D8\%AF\%D9\%85\%D8\%A7-\%D9\%84\%D8\%A7-

\%D9\%8A\%D8\%A8\%D9\%82\%D9\%89-

\%D9\%84\%D9\%84\%D8\%B3\%D9\%88\%D8\%B1\%D9\%8A\%D9\%8A\%D9\%86-

\%D8\%BA\%D9\%8A\%D8\%B1-\%D8\%A7\%D8\%B3\%D9\%85-

\%D8\%A7\%D9\%84\%D9\%84\%D9\%87/.

32. Mémoire créative de la révolution syrienne. Chroniques de la révolte syrienne:

Des lieux et des hommes 2011-2015. Presses de l’Ifpo, 2018.

https://doi.org/10.4000/books.ifpo.12765. p. 154.

33. Abouzeid, Rania. No Turning Back: Life, Loss, and Hope in Wartime Syria, 2019. http://www.worldcat.org/oclc/1098151277. p. 36.

34.

Muzâharat al-akfân fî bânyâs sûriyya 09/04/2011 [Manifestation des linceuls à Banyas Syrie 09/04/2011] :

Allfor Syria. مظاهرة الأكفان (1) في بانياس سوريا في 9. https://www.youtube.com/watch?v=6b8qbA9r3qo.. 35. Wensick, Arent Jan. « Takbīr ». Ecnyclopédie de l’Islam. Koninklijke Brill NV. Consulté le 5 janvier 2022. https://doi.org/10.1163/9789004206106 eifo_SIM_7330. 36. 
Ugarit Homs, hayy Hamîdiyya, 3 sawârîkh tanzal bi-l-qurb min al-musawwir 263 [Ugarit Homs quartier Hamidiyeh, 3 missiles tombent près du preneur d'image 26 3]

أوغاريت حمص حي الحميدية , Ugarit News - Syria. 263 Homs, شبكة أوغاريت الإخبارية - سوريا 3. https://www.youtube.com/watch? $\mathrm{v}=\mathrm{cBY7B3tBwnc}$.

Cette vidéo est décrite et analysée plus en détails par Cécile Boex « Témoignages filmés de la violence en Syrie. Le corps comme preuve, le récit comme réplique », dans Chehayed, Nibras. Images de chair et de sang: Penser le corps en Syrie (20112021). Cahiers de l'Ifpo 09. Beyrouth: Presses de l'Ifpo, 2021. http://www.worldcat.org/oclc/1286368952. $•$

37. On retrouve ce phénomène dans de nombreuses vidéos de bombardements. $\subseteq$ 38. Prédicateur salafiste originaire de Hama, Adnan al-Ar'our est parti en exil en Arabie-Saoudite suite aux événements de 1982. À partir de 2006, il s'est fait connaître pour ses prêches anti chiites diffusés par la chaîne saoudienne al-Safa. Au début de la révolte syrienne en 2011, il a pris fait et cause pour la rébellion et lancé sur la chaîne Wesal TV, une émission politico-religieuse appelée Avec la Syrie jusqu'à la victoire. Lors de l'une de ces émissions, il a divisé les alaouites en trois catégories : ceux qui ont soutenu l'opposition, ceux qui sont restés neutres, à qui rien ne sera fait, et ceux qui ont collaboré, « ceux-là, on les passera au hachoir et on donnera leur chair aux chiens », a-t-il lancé. Cet appel à la vengeance de la part d’un clerc populaire auprès des sunnites peu éduqués a particulièrement effrayé la communauté alaouite. $\leftrightarrows$

39.

Tawjîh al-Sheikh Adnan al-Ar'ur li-ahl al-sûriyya li-l-takbîr [Le Cheikh Adnan Ar'ur encourage les Syriens à faire le takbîr] :

AdnanAlarour. توجيه الشيخ عدنان العرعور لأهل سورية للتكبير, 2011.

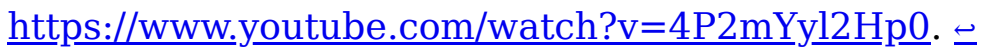

40. Al-Ar'ur qui s'est fait connaître comme téléprédicateur anti-chiite au milieu des années 2000 suit de près la politique iranienne, d'où probablement son appel à imiter les manifestants iraniens de 2009 qui en masse récitaient le takbir sur les toits à une heure précise de la nuit. 
41. Conversation Skype 12/11/2020.

42. Yazbik, Samar. 19 femmes: les Syriennes racontent. Paris, France: Stock, 2019. http://www.worldcat.org/oclc/1114114734.

43. Idem.

44. Ibid, p. 139-140.

45. Eylaf Bader Eddin, op. cit. p. 264.

46. Idem. $上$

47.

Hassan Hifar, «Al-harb fî suriyya : limâdha tahuddu shâbatun abnâ'a wa banâti jîlihâ 'ala 'adam al-injâb ? » [La guerre en Syrie : une jeune fille incite les autres jeunes à ne pas faire des enfants, pourquoi ?] :

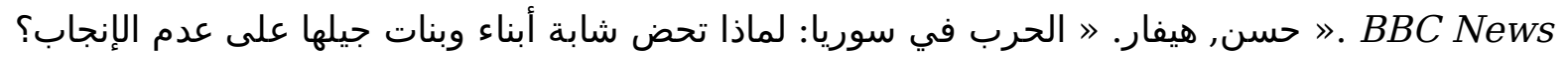
Consulté le 5 janvier 2022.

https://web.archive.org/web/20210403075746/https://www.bbc.com/arabic/middleeas $\underline{\mathrm{t}-56351010 .}$.

48. Sur les strategies de domestication et de forainisation (ou dépaysement), cf. Venuti, Lawrence. Translation Changes Everything: Theory and Practice. 0 éd. Routledge, 2012. https://doi.org/10.4324/9780203074428..

49. Yazbik, Samar, et Max Weiss. A woman in the crossfire: diaries of the Syrian revolution. London, Royaume-Uni de Grande-Bretagne et d'Irlande du Nord: Haus Pub., 2012. http://www.worldcat.org/oclc/835050758. p. 173-174.

50. Idem. 